\title{
CT Accuracy of Extrinsic Tongue Muscle Invasion in Oral Cavity Cancer
}

\author{
(D).C. Junn, (DK.L. Baugnon, (DE.A. Lacayo, DP.A. Hudgins, (DM.R. Patel, (DK.R. Magliocca, (D)A.S. Corey, (D) M. El-Deiry,
} (D).T. Wadsworth, (D).J. Beitler, (D)N.F. Saba, (D). Liu, and (D) A.H. Aiken

\begin{abstract}
BACKGROUND AND PURPOSE: Extrinsic tongue muscle invasion in oral cavity cancer upstages the primary tumor to a T4a. Despite this American Joint Committee on Cancer staging criterion, no studies have investigated the accuracy or prognostic importance of radiologic extrinsic tongue muscle invasion, the feasibility of standardizing extrinsic tongue muscle invasion reporting, or the degree of agreement across different disciplines: radiology, surgery, and pathology. The purpose of this study was to assess the agreement among radiology, surgery, and pathology for extrinsic tongue muscle invasion and to determine the imaging features most predictive of extrinsic tongue muscle invasion with surgical/pathologic confirmation.
\end{abstract}

MATERIALS AND METHODS: Thirty-three patients with untreated primary oral cavity cancer were included. Two head and neck radiologists, 3 otolaryngologists, and 1 pathologist prospectively evaluated extrinsic tongue muscle invasion.

RESULTS: Fourteen of 33 patients had radiologic extrinsic tongue muscle invasion; however, only 8 extrinsic tongue muscle invasions were confirmed intraoperatively. Pathologists were unable to determine extrinsic tongue muscle invasion in post-formalin-fixed samples. Radiologic extrinsic tongue muscle invasion had $100 \%$ sensitivity, $76 \%$ specificity, $57 \%$ positive predictive value, and $100 \%$ negative predictive value with concurrent surgical-pathologic evaluation of extrinsic tongue muscle invasion as the criterion standard. On further evaluation, the imaging characteristic most consistent with surgical-pathologic evaluation positive for extrinsic tongue muscle invasion was masslike enhancement.

CONCLUSIONS: Evaluation of extrinsic tongue muscle invasion is a subjective finding for all 3 disciplines. For radiology, masslike enhancement of extrinsic tongue muscle invasion most consistently corresponded to concurrent surgery/pathology evaluation positive for extrinsic tongue muscle invasion. Intraoperative surgical and pathologic evaluation should be encouraged to verify radiologic extrinsic tongue muscle invasion to minimize unnecessary upstaging. Because this process is not routine, imaging can add value by identifying those cases most suspicious for extrinsic tongue muscle invasion, thereby prompting this more detailed evaluation by surgeons and pathologists.

ABBREVIATIONS: $\mathrm{AJCC}=$ American Joint Committee on Cancer; $\mathrm{CECT}=$ contrast-enhanced $\mathrm{CT} ; \mathrm{ETM}=$ extrinsic tongue muscle; $\mathrm{ETMI}=$ extrinsic tongue muscle invasion; $\mathrm{FOM}=$ floor of the mouth; $\mathrm{OCC}=$ oral cavity cancer; $\mathrm{SCC}=$ squamous cell carcinoma

O ral cavity cancer accounts for nearly $30 \%$ of all head and neck malignancies. The diagnosis is often made at a more advanced stage, resulting in low 5-year survival rates: 50\%-60\% overall and as low as $22 \%$ for advanced-stage disease. ${ }^{1,2}$ Preoper-

Received June 21, 2016; accepted after revision August 30.

From the Departments of Imaging Sciences (J.C.J., K.L.B., P.A.H., A.S.C., A.H.A.), Otolaryngology (M.R.P., M.E.-D., J.T.W.), Pathology (K.R.M.), Radiation Oncology (J.J.B.), Hematology Oncology (N.F.S.), and Statistics (Y.L.), Emory University School of Medicine, Atlanta, Georgia; and Department of Radiology (E.A.L.), Georgetown University, Washington, DC.

Paper previously presented at: Annual Meeting of the Radiological Society of North America, November 29 to December, 2015; Chicago, Illinois.

Please address correspondence to Ashley H. Aiken, MD, 1364 Clifton Rd, Atlanta, GA 30322; e-mail: Ashley.aiken@emoryhealthcare.org

http://dx.doi.org/10.3174/ajnr.A4993 ative imaging provides the basis for staging and surgical planning for advanced oral cavity cancer (OCC). While the T1-T3 classification is based on tumor size, the T4 classification is based on identification of locally advanced disease with invasion of surrounding structures.

In the seventh edition of the American Joint Committee on Cancer (AJCC) staging, extrinsic tongue muscle invasion (ETMI) or bone involvement upstages the primary tumor to a T4a classification. ${ }^{3}$ Although all extrinsic tongue muscles (genioglossus, hyoglossus, palatoglossus, and styloglossus) are currently included in the AJCC staging system, only genioglossus and hyoglossus muscles are easily and routinely identified on cross-sectional imaging. The definitive determination of ETMI is often more difficult than bone invasion, but its presence also upstages 
to T4a. ETMI and upstaging a tumor may not only necessitate adjuvant radiation therapy but may also change the eligibility for enrollment in certain therapy clinical trials.

The current American College of Radiology guidelines advocate postoperative radiation therapy "in patients with higher-risk features for locoregional recurrence after surgery. These include advanced $\mathrm{T}$ stage (T3/T4), the presence of lymphovascular invasion, the presence of perineural invasion, positive surgical margins, lymph node involvement, extracapsular nodal extension, and bone involvement." ${ }^{\prime 4}$ For instance, postoperative radiation therapy would not be indicated for a patient with a T2N0 tumor with negative margins at surgery, no perineural invasion, or other high-risk factors. However, a size-matched OCC with similar characteristics staged T4a due to ETMI would require adjuvant radiation treatment or could influence the enrollment in current clinical trials that examine the role of adding systemic agents to radiation.

In current practice, head and neck radiologists evaluate invasion of at least the genioglossus muscle and, in many cases, the hyoglossus muscle. ${ }^{5-7}$ The styloglossus and palatoglossus muscles are more difficult to evaluate on imaging, though deep extension to involve the anterior tonsillar pillar would imply palatoglossus muscle invasion. Contrast-enhanced CT (CECT), MR imaging, and ultrasound can be used in OCC staging. ${ }^{8,9} \mathrm{MR}$ imaging is usually reserved for questions of perineural tumor or when extensive dental artifacts obscure oral cavity anatomy on CECT. While some authors favor MR imaging for $\mathrm{T}$ staging because of its soft-tissue resolution, recent studies have demonstrated the accuracy of CECT in the oral cavity. ${ }^{10-15}$ Proponents of CECT prefer this technique because of its accuracy in staging the primary tumor of the head and neck and its ability to combine with PET imaging for additional metabolic information regarding regional and distant metastases.

One of the shortcomings in evaluating the accuracy of radiologic ETMI is that there is no definite surgical or pathologic criterion standard to confirm invasion. Intraoperative evaluation and documentation of ETMI remains inconsistent without a set of guidelines. ETMI has not been consistently recorded within pathology reports at our institution; this inconsistency has created opportunities for inaccurate final pathologic staging and/or dependence on the radiologic stage. Currently, clinical and prognostic implications of ETMI in OCC remain elusive. ${ }^{16}$ Since the introduction of ETMI as a determinant for T4a disease in 1998 (3rd edition of the AJCC Manual for Staging of Cancer), no prospective radiologic-surgical-pathologic correlative studies have been performed to confirm the accuracy or prognostic importance of radiologic identification of ETMI. Boland et $\mathrm{al}^{17}$ questioned the prognostic implications and rationale behind automatic upstaging when only superficial extrinsic tongue muscles such as the hyoglossus and styloglossus are involved. Despite the lack of literature supporting the prognostic value and accuracy of radiologic ETMI, current practice often relies on radiologic staging for ETMI.

Although radiologic staging and determination of ETMI does not affect surgical eligibility, it can affect the postoperative treatment course, specifically the radiation treatment plan and eligibility for certain clinical trials. Because surgeons and pathologists do not routinely assess or report ETMI, the radiologic staging can "unofficially" persist despite the lack of data on CT accuracy. Therefore, our study investigates the feasibility of routine evalu- ation and reporting of ETMI across all 3 disciplines, in addition to the degree of agreement among radiology, surgery, and pathology to determine the accuracy of CECT for determining ETMI in cases of OCC.

\section{MATERIALS AND METHODS \\ Inclusion Criteria}

This institutional review board-approved prospective study accrued patients during a 12-month period. Before the initiation of this study, members of head and neck radiology, head and neck surgery, and head and neck subspecialty-designated pathology faculty agreed on consistent reporting of ETMI in radiology, operative, and pathology reports, respectively. After 1 year of prospectively recording ETMI, we searched the institutional radiology data base for untreated primary OCCs with preoperative CECT available for review and surgical resection at our institution. This search yielded 50 patients.

The inclusion criteria were the following:

1) Untreated OCC, accrued during the 12-month period

2) Preoperative CECT available for review

3) Surgical resection at our institution with operative and pathologic ETMI documentation.

Patients who did not undergo an operation at our institution and those who did not have intraoperative ETMI documentation were excluded from the study. Thirty-three patients met the inclusion criteria.

\section{Imaging Methods}

Patients underwent CECT on 1 of several commercially available CT systems with multidetector capability ranging from 16 to 64 channels. Onsite imaging CT studies were performed on LightSpeed VCT 64-section, Discovery HD 750, Discovery 16-section, and BrightSpeed 16-section scanners (GE Healthcare, Milwaukee, Wisconsin) or 16- to 64-channel scanners (Somatom Definition, Somatom Definition Flash; Siemens, Erlangen, Germany). Our split-bolus technique used $110 \mathrm{~mL}$ of intravenous iopamidol (Isovue-370; Bracco, Princeton, New Jersey), with $55 \mathrm{~mL}$ injected first at a rate of $2.5 \mathrm{~mL} / \mathrm{s}$, followed by a 40 -second delay, then another $55 \mathrm{~mL}$ at the same rate, with a total scan delay of 90 seconds, including a pre- and postcontrast saline bolus. We acquired contiguous axial images from the skull base through the mediastinum with the following settings: $1.25-\mathrm{mm}$ section thickness; pitch, 0.984:1; 0.7-second gantry rotation time; 25-cm FOV; $120 \mathrm{kV}$ (peak); and automatic exposure control with a noise index of 13.78. Reformatted images at 2.5-mm section thickness in the axial planes and $3-\mathrm{mm}$ sagittal and coronal reformations were sent to the PACS.

\section{Image Review}

Members of all 3 disciplines used a 4-point "Likert-type" scale to record an assessment of ETMI: "no" (definitely no ETMI), "probably no," "probably yes," and "yes" (definite ETMI). For radiology, 2 dedicated head and neck radiologists with Certificates of Added Qualification (10 years and 9 years of experience, respectively) blindly and independently reviewed these images. Discrepant cases were resolved by a consensus read. For each case, they recorded their overall impression regarding the presence of ETMI 


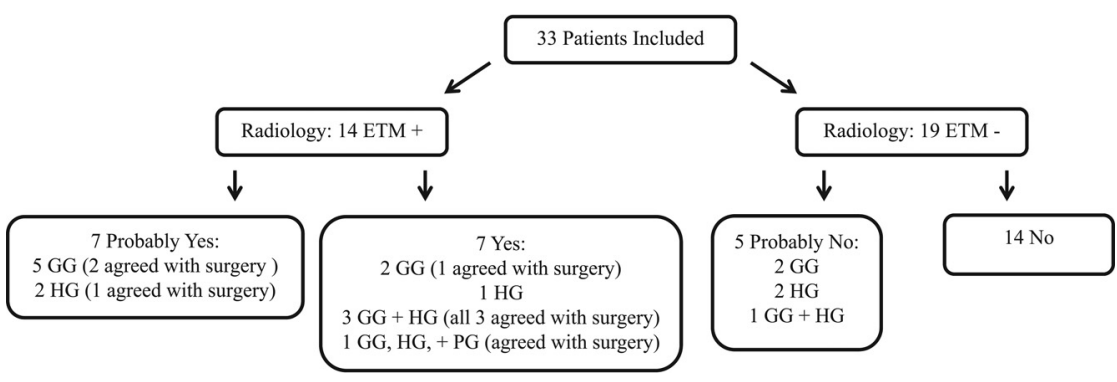

FIG 1. Schematic illustration of radiologic ETMI. Of the 33 included patients, 14 patients have radiologic ETMI. Of the 14 radiologic ETMI cases, 3 of 7 "probably yes" cases were concordant with surgery and 5 of 7 "yes" cases were concordant with surgery. GG indicates genioglossus; HG, hyoglossus; PG, palatoglossus.

Table 1: Radiologic and surgical assessment of ETMI

\begin{tabular}{lccc}
\hline Parameters & Radiologist 1 & Radiologist 2 & Surgery \\
\hline Yes & 6 & 7 & 7 \\
Probably yes & 7 & 7 & 1 \\
Probably no & 6 & 4 & 1 \\
No & 14 & 15 & 24 \\
\hline
\end{tabular}

a Individual ETMI responses by each radiologist and collective responses by head and neck surgeons.

but also evaluated each individual muscle: genioglossus, hyoglossus, styloglossus, and palatoglossus.

After the initial review, there were 5 imaging patterns: no contact, narrow contact, broad contact, linear enhancement within the muscle, and masslike enhancement within the muscle. "Narrow contact" was defined as $<50 \%$ of tumor contacting the extrinsic tongue muscle (ETM), and "broad contact" was defined as $>50 \%$ of the tumor contacting the ETM. After establishing these imaging features, the radiologists re-evaluated the cases and put each case into 1 of the 5 categories.

\section{Surgery Review}

One of 3 head and neck surgeons was always present during each operation, and an otolaryngology resident was routinely present to accurately record surgical ETMI prospectively. Before and during resection, the operating head and neck surgeon palpated the tumor en bloc for muscle tension anywhere between the proximal and distal insertion site to subjectively evaluate muscle invasion. Three of the muscles (genioglossus, styloglossus, and hyoglossus) originate from bone attachments, while the palatoglossus originates from the buccopharyngeal fascia. On completion of the resection, the operating surgeon carefully oriented the en bloc fresh specimen and evaluated the frozen section specimen in consultation with the pathologist performing gross examination. Any consensus evaluation during this intraoperative consultation was recorded under "surgery" for the purposes of this study.

\section{Pathology Review}

On completion of the intraoperative evaluation, the residual specimen was completely immersed in $10 \%$ buffered formalin. The formal pathology gross examination of the surgical specimen occurred on a formalin-fixed specimen within 1-2 days of receiving the specimen. During examination of the formalin-fixed specimen, a single head and neck pathology faculty member evaluated ETMI by macroscopic examination in cases lacking the interdisciplinary intraoperative notation of ETMI. The same pathologist retrospectively reviewed all finalized pathology reports and the corresponding hematoxylin-eosin-stained slides to ensure pathologic staging accuracy.

For statistical purposes, "probably yes" cases were included as "yes" and "probably no" cases were included as "no." Statistical analysis included calculation of the frequency of findings and the sensitivity, specificity, positive predictive value, and negative predictive value of radiologic assessment of ETMI by using surgery as the criterion standard. $\kappa$ statistics were used to assess interobserver reliability of radiologic ETMI determination.

\section{RESULTS}

This study recruited 50 consecutive patients with primary OCC during a 12-month period. Thirty-three patients met the inclusion criteria, with 9 women $(27 \%)$ and 24 men (73\%). Patient ages ranged from 31 to 88 years. Most of the subsites were the floor of the mouth (FOM) and oral tongue $(n=29)$, with only 5 cases from the alveolar ridge or buccal mucosa. The time from imaging to surgery ranged from 4 to 112 days, with an average imagingto-surgery time lapse of 36.3 days.

Of the 33 cases, 14 had radiologic findings of ETMI ( 7 yes and 7 probably yes). The 14 no and 5 probably no cases were all concordant with surgical findings.

Of the 7 yes cases, $2 / 7$ involved the genioglossus; $1 / 7$, the hyoglossus; $3 / 7$, the genioglossus and hyoglossus; and 1/7 the genioglossus, hyoglossus, and palatoglossus muscles. Of the 7 probably yes cases, there were 5 genioglossus and 2 hyoglossus involvements (Fig 1).

There were 8 surgical/gross pathologic ETMI cases during the routine intraoperative consultation for margin analysis (7 yes cases and 1 probably yes). Of the 7 radiologic yes cases, 5 agreed with surgery, and of the 7 radiologic probably yes cases, 3 were concordant with surgery (Fig 1). Due to tissue distortion and lack of orientation, it was not possible to evaluate ETMI in the delayed, post-formalin-fixed specimen in the postoperative setting.

The results of individual radiologist and surgical/gross pathologic determination of ETMI are represented in Table 1. These findings underscore the degree of uncertainty by radiologists when evaluating ETMI, as $40 \%$ of radiologist 1 and $33 \%$ of radiologist 2 findings fell under either the probably yes or probably no category. Despite the number of questionable cases, there was high interobserver agreement between the 2 radiologists: 0.937 . When cases with the original radiologic ETMI assessment were compared with surgical ETMI as the criterion standard, radiologic findings were $100 \%$ sensitive but specificity was only $76 \%$. The positive predictive value was $57.1 \%$, whereas the negative predictive value was $100 \%$. If surgery/pathology intraoperative evaluation was assumed to be the criterion standard, our study suggests that radiologic ETMI results in too many false-positives, especially if this finding leads to additional treatment and is not confirmed at the time of the operation.

Subsequently, the radiologists re-reviewed, without knowledge of surgical or pathologic findings with respect to ETMI, all 
Table 2: Detailed review of specific imaging features ${ }^{a}$

\begin{tabular}{|c|c|c|}
\hline $\begin{array}{l}\text { Initial Radiologic } \\
\text { Assessment }\end{array}$ & Radiologic Features on Reassessment & Surgical Finding \\
\hline \multicolumn{3}{|l|}{7 Probably yes } \\
\hline $\mathrm{GG}^{\mathrm{b}}$ & Masslike enhancement & GG \\
\hline $\mathrm{GG}^{\mathrm{b}}$ & $\begin{array}{l}\text { Broad contact (some borders not visualized } \\
\text { due to amalgam) }\end{array}$ & $\begin{array}{l}\text { ETMI without } \\
\text { specification }\end{array}$ \\
\hline GG & Narrow contact & None \\
\hline GG & Narrow contact & None \\
\hline GG & Narrow contact & None \\
\hline HG & Linear enhancement & None \\
\hline$H G^{b}$ & $\begin{array}{l}\text { Linear enhancement, also not definitive } \\
\text { intraoperatively }\end{array}$ & HG (probably yes) \\
\hline \multicolumn{3}{|c|}{ (1) } \\
\hline$G^{b}$ & Masslike enhancement & GG HG \\
\hline GG & Broad contact & None \\
\hline HG & Narrow contact & None \\
\hline GG HG ${ }^{b}$ & Masslike enhancement & GG HG \\
\hline GG HG & Masslike enhancement & GG HG \\
\hline GG HG ${ }^{b}$ & Masslike enhancement & GG HG \\
\hline GG HG PG ${ }^{b}$ & Masslike enhancement & GG HG PG \\
\hline \multicolumn{3}{|l|}{5 Probably no } \\
\hline$G^{b}$ & Narrow contact & None \\
\hline $\mathrm{GG}^{\mathrm{b}}$ & Broad contact (partially obscured by amalgam) & None \\
\hline$H G^{b}$ & Linear enhancement & None \\
\hline $\mathrm{HG}^{\mathrm{b}}$ & Linear enhancement & None \\
\hline GG HG ${ }^{b}$ & Narrow contact & None \\
\hline
\end{tabular}

Note:-GG indicates genioglossus; HG, hyoglossus; PG, palatoglossus.

a Detailed list of the imaging features in the 19 cases with initial "probably yes," "yes," and "probably no" classifications. ${ }^{\mathrm{b}}$ Agree with operational findings.

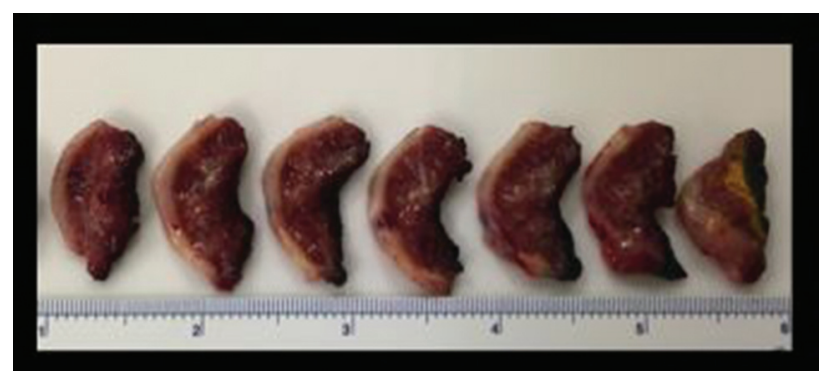

FIG 2. Partial glossectomy specimen entirely sectioned. Once formalin-fixed, ETMI or subtle tongue muscle anatomy are nearly impossible to precisely identify.

cases to determine radiologic features most predictive of ETMI to establish whether more specific imaging features could improve accuracy. For all cases scored "no" for ETMI, the tumor had no contact with any extrinsic tongue muscle. For cases scored probably no, probably yes, or yes, there were 4 imaging patterns: masslike enhancement, linear enhancement, broad contact, or narrow contact (Table 2). Masslike enhancement was the only imaging feature that accurately predicted surgical/gross pathologic ETMI (100\% specificity, $75 \%$ sensitivity, $100 \%$ positive predictive value, and $93 \%$ negative predictive value). Narrow contact alone never predicted (100\% negative predictive value) surgical/gross pathologic ETMI. Broad contact and linear enhancement were less reliable with $33 \%$ and $25 \%$ positive predictive values, respectively.

\section{DISCUSSION}

As we continue to advance the role of imaging in the staging of head and neck cancer, it is important to review and understand the implications and accuracy in a multidisciplinary setting. On our multidisciplinary tumor board, the oncologists and radiation oncologists often request that head and neck radiologists prospectively determine ETMI on imaging, regardless of pathologic and surgical confirmation. After discussing ETMI as a group, we learned that not only are pathologists not trained to routinely look for ETMI but it is also not possible to accurately determine ETMI retrospectively on a formalin-fixed specimen. Furthermore, surgeons also did not routinely record ETMI. Representatives from radiology, surgery, and pathology decided to prospectively look closely for ETMI for this study.

\section{Multidisciplinary ETMI Evaluation}

This prospective study proposes a more standardized approach for multidisciplinary evaluation and documentation of ETMI. Our findings highlight the importance of a true multidisciplinary team to coordinate the effort of radiologists, surgeons, and pathologists. We have initiated a sequence of events for the most accurate and optimal OCC staging based on ETMI. First, the radiologist evaluates and documents ETMI on the preoperative imaging examination. Then the surgeon performs a primary resection in most cases. At the time of the operation, the surgeon subjectively evaluates ETMI by palpating the tumor and observing tension on the muscle insertions. After the en bloc resection, the specimen is simultaneously evaluated in the frozen section suite by the surgeon and the pathology team. The suspicion or presence of ETMI or both are documented on the fresh specimen, before formalin fixation.

We have found that collaborative intraoperative evaluation by the surgeons and pathologists is the best opportunity to identify ETMI. The ETMs are identified when the specimen is fresh; then, serial cuts are made through the tumor to evaluate specific muscle invasion grossly, which is subsequently confirmed by microscopic examination. On completion of the intraoperative pathology evaluation, fresh resection specimens immersed in formalin will then be macrodissected to capture key relationships between tumor and resected normal anatomic structures and to evaluate any remaining surgical margins. Microscopic examination of these selected sections provides the basis of the final pathology report. However, retrospective determination of ETMI after formalin fixation alone is challenging or, in most cases, impossible due to tissue fixation/distortion, muscle retraction, and inability to identify a small portion of the ETM on a completely sectioned specimen (Fig 2).

\section{ETMI Imaging Features}

When we reassessed all of the probably yes, yes, and probably no cases, we discovered 4 different imaging patterns: masslike enhancement, narrow contact, broad contact, and linear enhance- 

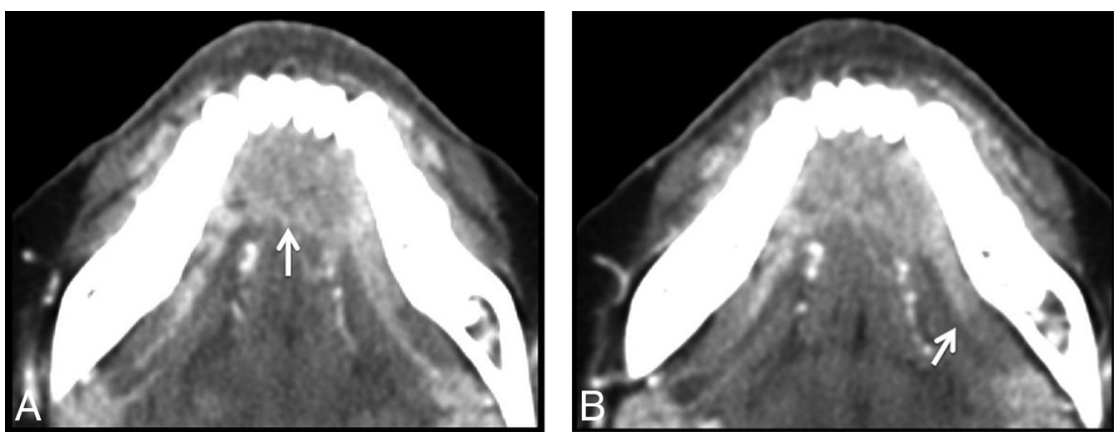

FIG 3. A 70-year-old woman with anterior FOM squamous cell carcinoma (SCC), pT4aNOMO. Concordant: Radiology and surgery positive for ETMI (genioglossus). A, Anterior floor of the mouth tumor with masslike invasion into the genioglossus muscle at its insertion site (arrow). B, Dilated left submandibular duct lateral to the hyoglossus muscle (arrow).
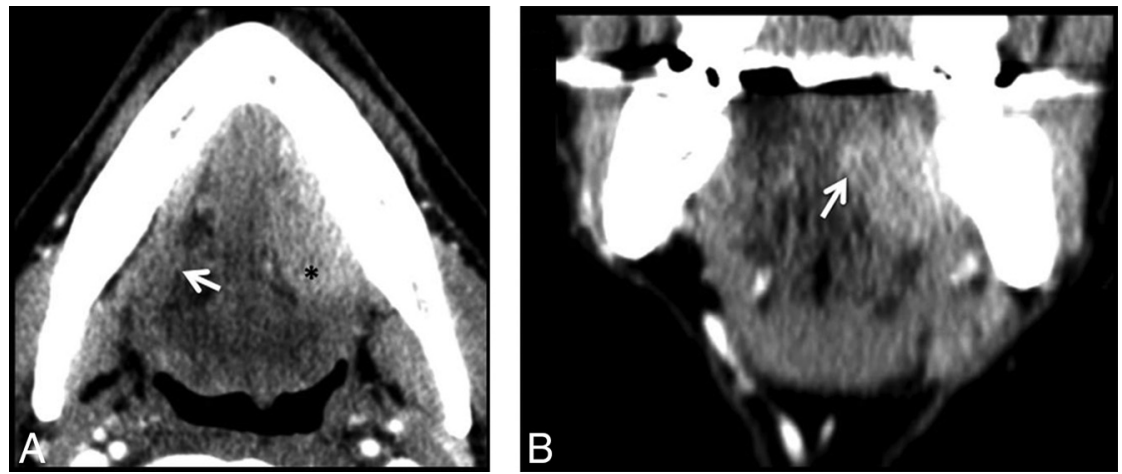

FIG 4. A 53-year-old man with left FOM SCC, pT4aN2cM0. Concordant: Radiology and surgery positive for ETMI (genioglossus and hyoglossus muscles). A, Axial image shows broad contact along the genioglossus muscle with obliteration and masslike enhancement in the expected location of the hyoglossus muscle (asterisk). Note the uninvolved hyoglossus muscle on the contralateral side (arrow). B, Coronal image shows complete replacement of the hyoglossus muscle with tumor and masslike invasion into the genioglossus muscle (arrow).
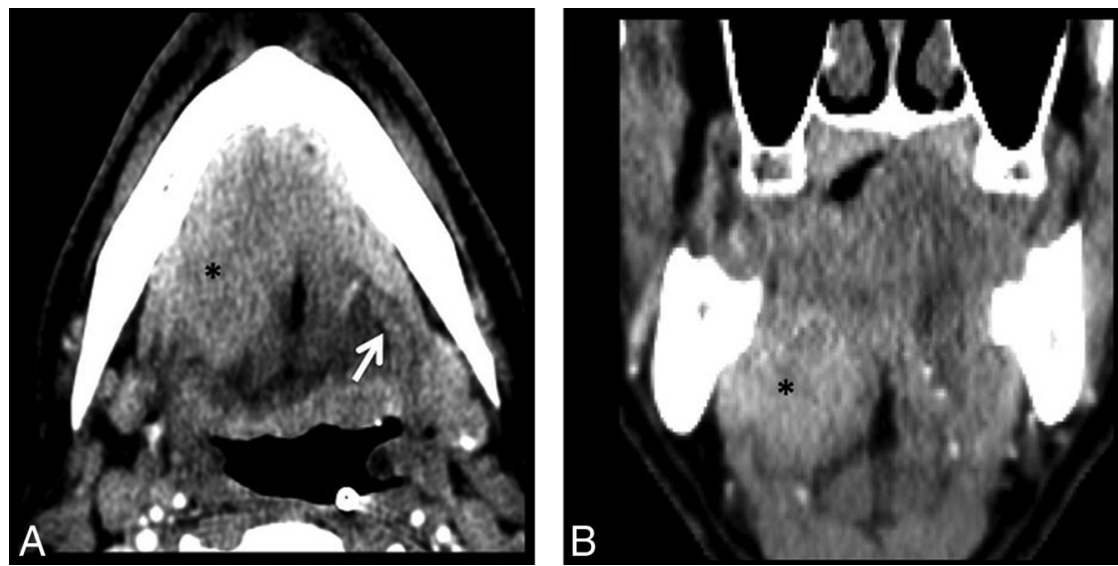

FIG 5. A 56-year-old man with right FOM SCC, pT4aN2bM0. Concordant: Radiology and surgery positive for ETMI (genioglossus and hyoglossus muscles). A, Axial image shows masslike enhancement involving the entire genioglossus and hyoglossus muscles (asterisk). A normal hyoglossus muscle is seen on the contralateral side (arrow). B, The coronal image confirms masslike tumor enhancement involving the genioglossus and hyoglossus muscles (asterisk).

ment. All 6 patients with masslike enhancement also had ETMI on surgical/pathologic intraoperative evaluation. None of the 6 patients with narrow contact had surgical/pathologic ETMI. Only 1 of 3 patients with radiologic broad contact had surgical/gross pathologic ETMI. Similarly, only 1 of 4 patients with linear enhancement had surgical/pathologic ETMI.
Therefore, masslike enhancement indistinguishable from the ETM is the most specific finding that leads to an agreement among radiology, surgery, and gross pathology (Figs 3-5). Linear enhancement (Fig 6) and broad contact are less reliable imaging signs with variable intraoperative findings. On the basis of our results, narrow contact (Figs 7 and 8) does not predict surgical/pathologic ETMI and should not be used to determine ETMI. Of the 6 radiologic ETMI cases without surgical ETMI, 1 patient had linear enhancement, 4 patients had anterior floor of the mouth tumor near the genioglossus genial tubercle insertion site with only narrow contact, and 1 patient had a large oral tumor mass above the genioglossus muscle with broad contact.

\section{Limitations in Other Disciplines}

Surgical evaluation is imprecise for determining ETMI. Intraoperative assessment by surgical palpation alone is a subjective evaluation and, in our experience, is not consistently documented in the operative report. Additionally, there is often not a definitive answer regarding invasion of the small, thin hyoglossus muscle because it may not be readily visible to the surgeons intraoperatively. In turn, hyoglossus invasion is not discernible by the pathologist. Therefore, to optimize evaluation of ETM, a careful and deliberate "consensus" examination and analysis of the fresh resection specimen by the surgeon and pathology team at the time of intraoperative consultation, and before formalin fixation, appear to result in the highest level of confidence among the surgeons and pathologists and should be the criterion standard.

Although documentation of pathologic ETMI is important, ${ }^{18}$ a review of the current pathology dissection texts and literature reveals no systematic approach to ETMI determination in the fresh or formalin-fixed state. ${ }^{19,20}$ While this study highlights a mechanism to facilitate identification of ETMI, particularly in collaboration with surgical colleagues, variations in intraoperative and interdisciplinary work flow and resources may preclude replication at some institutions. In cases in which portions of the oral tongue are resected from their bony attachments, the surrounding anatomic context and relationships can be difficult to discern in a formalin-fixed specimen because they may have been multiply-sectioned or key por- 
tions of the specimen may have been removed for frozen section analysis intraoperatively. We believe this scenario represents a hurdle in providing microscopic confirmation of ETMI. Other obstacles to pathologic confirmation of ETMI include the following: the relatively nonspecific microscopic appearance of intrinsic tongue skeletal muscle in comparison with extrinsic tongue muscle, the procedures performed to evaluate tumor margin status intraoperatively, piecemeal submission of subsequent portions of tissue/margins (which may or may not include ETM), distortion induced by formalin fixation, the range of expertise or familiarity of the person performing gross examination with an oral resection specimen, and the appreciation of the importance of ETMI by the pathologist performing gross examination. Furthermore, it seems impossible to expect pathologic confirmation of hyoglossus invasion once the muscle is detached from insertions because it is too small for routine identification.

All specialties need to be aware, therefore, that hyoglossus muscle invasion appears, on the basis of our results, to be an observation that might be best made on cross-sectional imaging when there is masslike enhancement in the muscle.
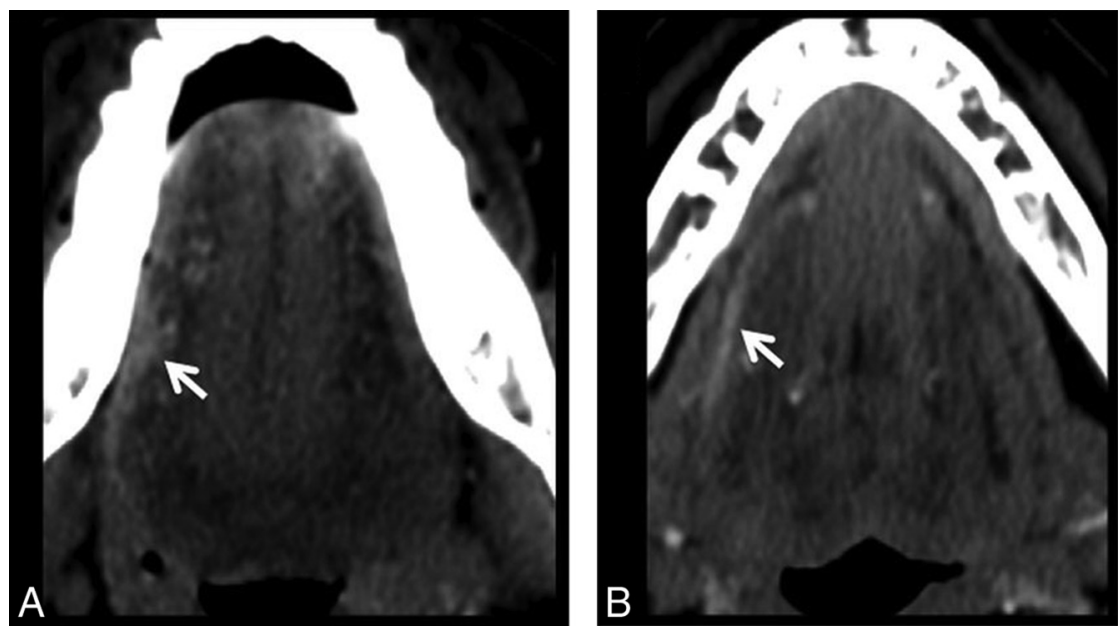

FIG 6. A 33-year-old woman with right lateral tongue SCC, PTINOMO. Discordant: Radiology positive for ETMI (hyoglossus muscle) and surgery negative. Initial radiologic evaluation was probably yes due to linear enhancement along the hyoglossus muscle. A, Small right lateral tongue mass (arrow). B, Linear enhancement of the hyoglossus muscle (arrow).
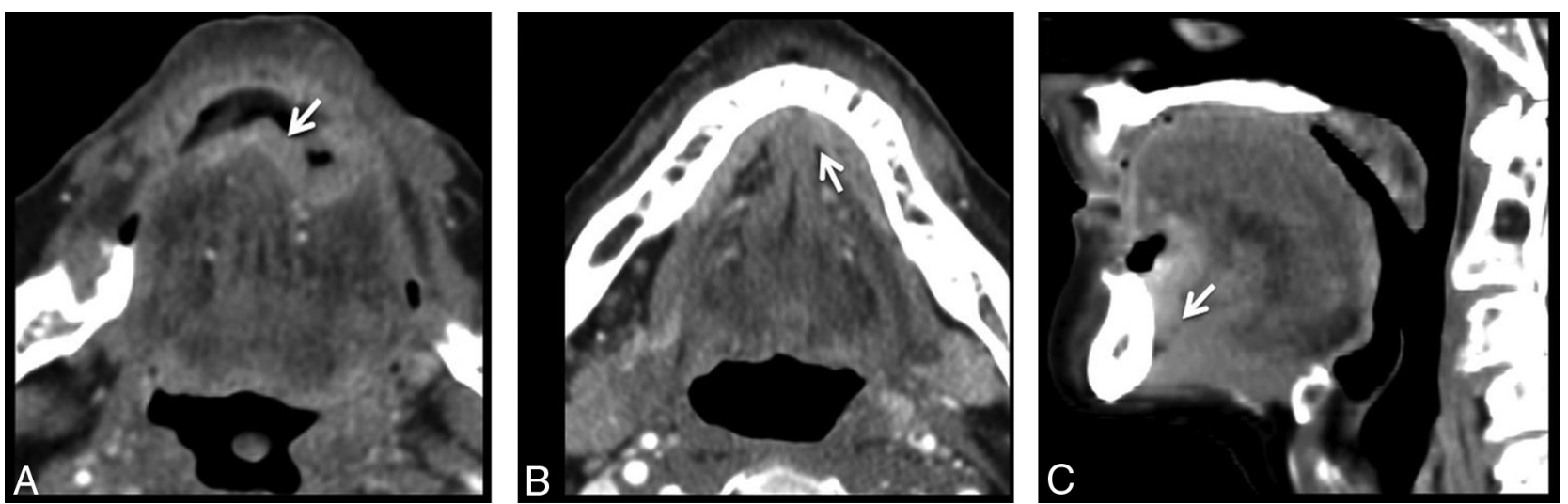

FIG 7. A 55-year-old woman with anterior FOM SCC, pT2NOMO. Discordant: Radiology positive (genioglossus muscle) and surgery negative for ETMI. $A$ and $B$, Axial images show an anterior FOM tumor with apparent broad contact at the genioglossus insertion site (arrow). C, A closer look at the axial and sagittal images shows the tumor just above the insertion site with only narrow contact at the superior margin of the genioglossus insertion (arrow). This finding highlights a possible pitfall of the axial acquisition because volume averaging could depict the tumor with more masslike involvement of the ETM. 


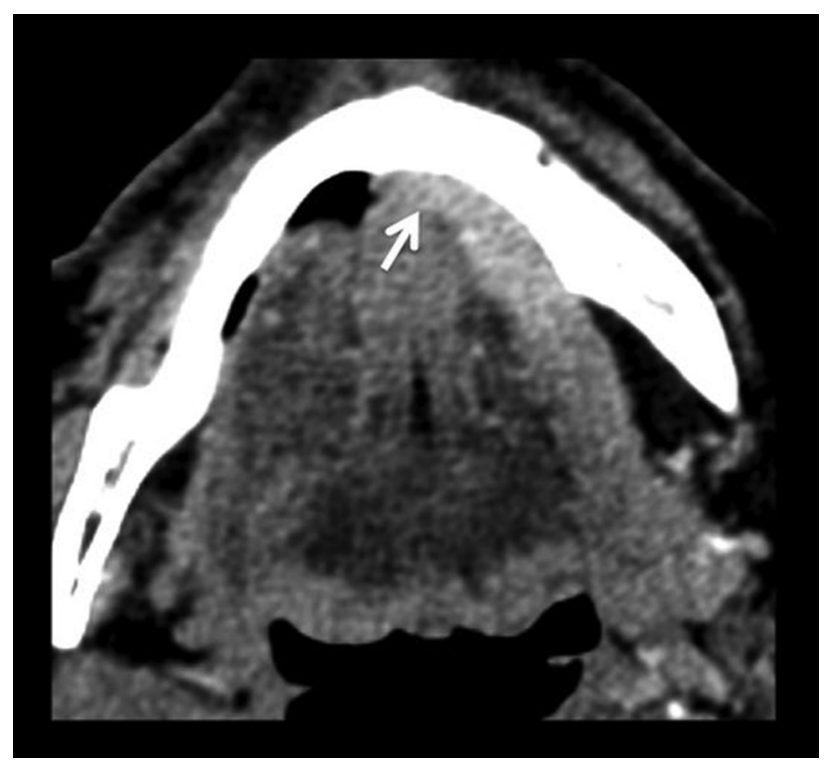

FIG 8. A 78-year-old man with left FOM SCC, pT4aNOMO. Note that $\mathrm{T} 4 \mathrm{a}$ is for pathologic bone invasion only. Discordant: Radiology positive (genioglossus muscle) and surgery negative for ETMI. This was initially scored as probably yes due to broad contact, but in retrospect, there appears to be only focal contact at the insertion point of the left genioglossus muscle (arrow).

play a role in evaluating equivocal cases, given its greater softtissue detail.

The limitations of this study include the small sample size and multiple different surgeons and pathologists involved in the surgical determination of ETMI, though we attempted to minimize this limitation by meeting prospectively to standardize our approach. The high interobserver agreement regarding radiologic ETMI determination is likely because both readers are subspecialty trained neuroradiologists with a focus on head and neck imaging.

\section{CONCLUSIONS}

CECT is sensitive for ETMI but has reduced specificity (76\%) compared with intraoperative surgical and pathology consensus evaluation. Masslike enhancement is the most specific imaging feature for predicting ETMI. Broad contact and linear enhancement of ETM are equivocal and often lead to false-positives; and narrow contact is not predictive.

A careful but perhaps time-intensive consensus read between the operating surgeon and the pathologist performing gross examination (in which the surgeon properly orients the fresh specimen for the pathologist) should be the criterion standard for ETMI. Because this process is currently not standard practice, imaging can best add value by identifying those cases most suspicious for ETMI, thereby prompting a more detailed evaluation by the surgeons and pathologists in these cases. This preliminary study has established imaging features that are more predictive of ETMI and will facilitate future correlation with patient outcomes to determine whether radiologic ETMI is indeed prognostic.

Disclosures: Mihir R. Patel—UNRELATED: Consultancy: AstraZeneca, Comments: served on a panel discussing potential genes and markers of immunotherapy, specifically as it relates to Programmed Death Ligand-1. Amanda S. Corey-UNRELATED: Consultancy: Radiology in Medical Development, Comments: Reviewer; Employment: Emory University Hospital and Clinics; Payment for Lectures including Service on Speakers Bureaus: Osler Institute, Comments: lecturer. Nabil F. Saba_UNRELATED: Consultancy:
Bristol-Myers Squibb, Merck, Pfizer, Comments: consultation fees; Royalties: PubMed publications. Yuan Liu-UNRELATED: Grant: National Institutes of Health/National Cancer Institute, Comments: award No. P30CA138292.* *Money paid to the institution.

\section{REFERENCES}

1. Jemal A, Thun MJ, Ries LA, et al. Annual report to the nation on the status of cancer, 1975-2005, featuring trends in lung cancer, tobacco use, and tobacco control. J Natl Cancer Inst 2008;100:1672-94 CrossRef Medline

2. Steele TO, Meyers A. Early detection of premalignant lesions and oral cancer. Otolaryngol Clin North Am 2011;44:221-29, vii CrossRef Medline

3. Edge SB, Compton CC. The American Joint Committee on Cancer: the 7 th edition of the AJCC cancer staging manual and the future of TNM. Ann Surg Oncol 2010;17:1471-74 CrossRef Medline

4. Salama JK, Saba N, Quon H, et al; Expert Panel on Radiation Oncology-Head and Neck. ACR appropriateness criteria ${ }^{\circledR}$ adjuvant therapy for resected squamous cell carcinoma of the head and neck. Oral Oncol 2011;47:554-59 CrossRef Medline

5. Aiken AH. Pitfalls in the staging of cancer of oral cavity cancer. Neuroimaging Clin N Am 2013;23:27-45 CrossRef Medline

6. Kirsch C. Oral cavity cancer. Top Magn Reson Imaging 2007;18: 269-80 CrossRef Medline

7. Chong V. Oral cavity cancer. Cancer Imaging 2005;5(Spec No A): S49-52 CrossRef Medline

8. Law CP, Chandra RV, Hoang JK, et al. Imaging the oral cavity: key concepts for the radiologist. Br J Radiol 2011;84:944-57 CrossRef Medline

9. Yuen AP, Ng RW, Lam PK, et al. Preoperative measurement of tumor thickness of oral tongue carcinoma with intraoral ultrasonography. Head Neck 2008;30:230-34 CrossRef Medline

10. Arya S, Rane P, Deshmukh A. Oral cavity squamous cell carcinoma: role of pretreatment imaging and its influence on management. Clin Radiol 2014;69:916-30 CrossRef Medline

11. Dammann F, Horger M, Mueller-Berg M, et al. Rational diagnosis of squamous cell carcinoma of the head and neck region: comparative evaluation of CT, MRI, and 18FDG PET. AJR Am J Roentgenol 2005; 184:1326-31 CrossRef Medline

12. Park JO, Jung SL, Joo YH, et al. Diagnostic accuracy of magnetic resonance imaging (MRI) in the assessment of tumor invasion depth in oral/oropharyngeal cancer. Oral Oncol 2011;47:381-86 CrossRef Medline

13. Lam P, Au-Yeung KM, Cheng PW, et al. Correlating MRI and histologic tumor thickness in the assessment of oral tongue cancer. AJR Am J Roentgenol 2004;182:803-08 CrossRef Medline

14. Gődény M. Prognostic factors in advanced pharyngeal and oral cavity cancer; significance of multimodality imaging in terms of 7th edition of TNM. Cancer Imaging 2014;14:1 CrossRef Medline

15. Madana J, Laliberté F, Morand GB, et al. Computerized tomography based tumor-thickness measurement is useful to predict postoperative pathological tumor thickness in oral tongue squamous cell carcinoma. J Otolaryngol Head Neck Surg 2015;44:49 CrossRef Medline

16. Boland PW, Pataridis K, Eley KA, et al. A detailed anatomical assessment of the lateral tongue extrinsic musculature, and proximity to the tongue mucosal surface: does this confirm the current TNMT4a muscular subclassification? Surg Radiol Anat 2013;35:559-64 CrossRef Medline

17. Boland PW, Pataridis K, Eley KA, et al. Automatic upstaging of tongue squamous cell carcinoma with lateral extrinsic muscle involvement is not justified. Int J Oral Maxillofac Surg 2013;42:1397402 CrossRef Medline

18. Zarbo RJ, Barnes L, Crissman JD, et al. Recommendations for the reporting of specimens containing oral cavity and oropharynx neoplasms: Association of Directors of Anatomic and Surgical Pathology. Hum Pathol 2000;31:1191-93 CrossRef Medline

19. Lester S. Manual of Surgical Pathology: Expert Consult. Philadelphia: Elsevier; 2010:473-86

20. Westra H, Hruban RH, Phelps TH, et al. Surgical Pathology Dissection: An Illustrated Guide. New York: Springer; 2003:44-47 